\title{
Clinical features and management of organic acidemias in Japan
}

\author{
Daisuke Fujisawa $^{1}$, Kimitoshi Nakamura ${ }^{1}$, Hiroshi Mitsubuchi ${ }^{1}$, Toshihiro Ohura ${ }^{2}$, Yosuke Shigematsu ${ }^{3}$, \\ Tohru Yorifuji ${ }^{4}$, Mureo Kasahara ${ }^{5}$, Reiko Horikawa ${ }^{6}$ and Fumio Endo ${ }^{1}$
}

Organic acidemias (OAs) are rare inborn errors of metabolism. The clinical presentations of methylmalonic acidemia (MMA) and propionic acidemia (PA) in Japan have not yet been examined in detail. We aimed to investigate the clinical presentations of OAs in Japan and evaluate current therapies for improving long-term outcomes, especially in MMA and PA cases.

Questionnaires were sent to 928 institutions in 2009 inquiring about OAs, and secondary questionnaires were sent to those who confirmed that they had diagnosed and/or treated such cases; 119 cases were eventually included for analysis. In Japan, the majority of OAs was MMA, which was associated with a high mortality rate. The survival rates at 20 years of age in vitamin B12-unresponsive MMA, vitamin B12-responsive MMA and PA patients were $69.8 \%, 94.4 \%$ and $95.8 \%$, respectively. Factors associated with mortality in MMA were failure to thrive, hypoglycemia and pancreatitis. Factors associated with mental retardation in vitamin B12-unresponsive MMA, vitamin B12-responsive MMA, and PA were seizure and liver dysfunction, seizure and failure to thrive, and failure to thrive, respectively. We advocated that avoiding failure to thrive due to too restricted protein diet, hypoglycemia and pancreatitis associated with mortality lead to improve outcome, especially in vitamin B12unresponsive MMA patients.

Journal of Human Genetics (2013) 58, 769-774; doi:10.1038/jhg.2013.97; published online 26 September 2013

Keywords: methylmalonic academia; organic acidemias; propionic acidemia

\section{INTRODUCTION}

Organic acidemias (OAs) are rare inborn errors of metabolism that can be life-threatening and have severe complications. ${ }^{1}$ In Japan, studies have focused on the long-term outcomes of inherited metabolic diseases, such as urea cycle disorders and glycogen storage disease. ${ }^{2,3}$ As OAs usually present with common symptoms due to metabolic acidosis, early diagnosis and appropriate treatment are essential for obtaining optimal outcomes. In the diagnosis of OAs, gas chromatography mass spectrometry (GC/MS) is typically used to analyze urinary organic acids. Furthermore, in recent years, tandem mass spectrometry (MS/MS) has also become available for newborn screening (NBS) and advanced metabolic tests, which are performed as indicated based on family history and/or routine laboratory test results. ${ }^{4-8}$

Methylmalonic acidemia (MMA) and propionic acidemia (PA) are the most frequent types of organic acid metabolism disorders and are inherited in an autosomal recessive pattern. These conditions are caused by severe inborn errors of catabolism of the amino acids isoleucine, valine, methionine and threonine, as well as metabolism of odd-chain fatty acids and cholesterol side chains. MMA is characterized by an abnormal accumulation of methylmalonyl-CoA and methylmalonic acid. It is caused by a defect of the methylmalonyl-CoA mutase (MCM) or a defect in the synthesis of $5^{\prime}$-deoxyadenosylcobalamin, which is the cofactor of MCM (cblA, cblB, cblD). ${ }^{1}$ Moreover, some disorders involving methylcobalamin metabolism result in MMA and homocysteinuria (cblC, cblD, cblF). The deficiencies of MCM are subdivided into mut ${ }^{0}$, with complete loss of MCM activity, and mut $^{-}$, with partial residual activity. Clinically, vitamin B12responsive MMA is considered when a patient with MMA responds effectively to vitamin B12 supplementation and exhibits a defective synthesis of cobalamin and the presence of a part of mut $^{-}$. In contrast, vitamin B12-unresponsive MMA is considered when a patient with MMA responds poorly to vitamin B12 supplementation and exhibits the presence of mut $^{0}$ or a residual part of mut ${ }^{-}$. PA is characterized by an abnormal accumulation of propionic acid and metabolites such as methylcitrate, 3-hydroxypropionic acid and propionyl glycine. It is caused by a defect of the enzyme propionyl-CoA carboxylase (PCC), which converts propionyl-CoA to methylmalonyl-CoA.

The clinical presentations of MMA and PA can be similar in many ways. Most patients with the early-onset type present in the neonatal

${ }^{1}$ Department of Pediatrics, Kumamoto University Graduate School of Medical Sciences, Kumamoto, Japan; ${ }^{2}$ Division of Pediatrics, Sendai City Hospital, Sendai, Japan; ${ }^{3}$ School of Nursing, Faculty of Medical Sciences, University of Fukui, Fukui, Japan; ${ }^{4}$ Department of Pediatric Endocrinology and Metabolism, Children's Medical Center, Osaka City General Hospital, Osaka, Japan; ${ }^{5}$ Department of Transplantation Surgery, National Center for Child Health and Development, Tokyo, Japan and ${ }^{6}$ Department of Endocrinology and Metabolism, National Center for Child Health and Development, Tokyo, Japan

Correspondence: Dr K Nakamura, Department of Pediatrics, Kumamoto University Graduate School of Medical Sciences, 1-1-1 Honjo, Kumamoto 860-8556, Japan. E-mail: nakamura@kumamoto-u.ac.jp

Received 29 April 2013; revised 22 August 2013; accepted 23 August 2013; published online 26 September 2013 
period, within 1 month after birth, with nonspecific signs such as appetite loss, recurrent vomiting, lethargy, hypotonia and tachypnea. Some patients have more severe complications such as hyperammonemia, seizure, deep coma and death. Patients with the late-onset type have similar clinical pictures as the early-onset type and can be diagnosed with failure to thrive and/or developmental delay. Regardless of the time of onset, decompensation, manifesting as impaired consciousness, can be life-threatening and indicates a severe clinical condition. ${ }^{5}$

Treatment options include a restricted protein diet to reduce the levels of harmful amino acids in affected patients. Moreover, administration of antibiotics can result in a reduction in the propionate produced from gut bacteria. Arginine and sodium benzoate are also prescribed to treat hyperammonemia. Hemodialysis must be implemented without delay. Carnitine supplementation is effective to release trapped CoA and prevent secondary carnitine deficiency. ${ }^{9}$ However, as there is no specific therapy for MMA and PA, patients who recover from decompensation often experience neurologic sequelae. In the present study, we aimed to investigate the clinical presentations of organic acidemias in Japan and evaluate current therapies for improving long-term outcome, especially in cases with MMA and PA.

\section{MATERIALS AND METHODS}

This study was approved by the Ethics Committee of the Faculty of Life Science, Kumamoto University. In 2009, a questionnaire was sent to 928 institutions, including the Departments of Pediatrics, Neonatology, Genetics, and Transplant surgery, asking doctors if they diagnosed or provided medical care to OAs patients. In response, 201 institutions confirmed that they had diagnosed and/or treated inborn errors of metabolism. A secondary detailed questionnaire concerning OAs was then sent to these 201 institutions, of which 41 responded. In 2012, we conducted follow-up research to reveal outcome. In total, 119 cases of OAs were collected. The definition of clinical manifestations of OAs used in this study was the same as that previously described. ${ }^{3}$ In addition, mental retardation was diagnosed if the patient's intelligence quotient was $<70$, in standardized tests, such as the Wechsler Intelligence Scale for Children and the Wechsler Adult Intelligence Scale.

\section{Statistical analysis}

Data were analyzed using IBM SPSS statistics 21.0. Descriptive and inferential statistics were used in this study. Significance of differences was assessed using the Mann-Whitney $U$-test or Kruskal-Wallis test. For data with small values, the Fisher exact test was used for a more precise $P$-value. Independence was tested using the chi-square test. Mortality rates were compared using KaplanMeier analysis with the logrank test.

\section{RESULTS}

\section{General characteristics}

Characteristics of affected individuals were shown in Table 1. There were 17 deaths, including 10 patients with vitamin B12-unresponsive MMA (58.8\%), 2 with vitamin B12-responsive MMA (11.8\%) and 1 with MMA whose response to vitamin B12 was unknown (Table 1). The deceased patients comprised 13 of 65 MMA patients and 2 of 30 PA patients. There was statistical difference in survival rates. In 2012, at a subsequent follow-up, the median age of all patients with OAs was 128 months (interquartile range (IQR), 68.3-191.8). There was no significant difference in age distribution between MMA and PA patients. The median age of the 17 deceased patients was 11 months (IQR, 11-84 months; range, 5 days to 23 years). There was also no significant difference in sex between MMA and PA patients. The number of all male OA patients was $55(46 \%)$. In Table 2, characteristics of onset and diagnosis of OAs were shown.
Table 1 Characteristic of affected individuals

N $(\%)$

Death case $(\%)$

\section{Methylmalonic acidemia}

Vitamin B12-unresponsive

$42(35.2) \quad 10(58.8)$

Vitamin B12-responsive

Unidentified on response to vitamin B12

$20(16.8) \quad 2(11.8)$

Propionic acidemia

Holocarboxylase synthetase (HCS) deficiency

Glutaric aciduria type II

$3(2.51) \quad 1(5.9)$

$30(25.1) \quad 2(11.8)$

Isovalericacidemia

3-Methylcrotnyl-CoA carboxylase (3MCC) deficiency

Glutaric aciduria type

$6(5.0)$

$5(4.0)$

$2(11.8)$

$3(2.5)$

$3(2.5)$

$2(1.7)$

$2(1.7)$

$1(0.8)$

Ethylmalonic aciduria

$1(0.8)$

$\beta$-Ketotiolase deficiency

$1(0.8)$

3-Hydroxy-3-methylglutaric aciduria

119

1 (5.9)

-

1 (5.9)

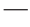

Table 2 Characteristics of onset and diagnosis of OAs

(a) Onset

First month of life $\quad 48$ (44)

1 month-1 year 34 (31)

Older than 1 year $13(12)$

Total

(b) Diagnosis

Within a month $72(74)$

1 month-1 year $19(20)$

Over 1 year 6 (6)

Total

aFifteen in 110 cases did not have an onset and were diagnosed by newborn screening or advanced metabolic tests.

NBS by MS/MS and advanced metabolic tests were used to diagnose 11 and 4 cases, respectively (vitamin B12-responsive MMA, 1 and 3 cases; vitamin B12-unresponsive MMA, 1 and 0 case; PA, 7 and 1 cases; HCS deficiency, 1 and 0 case; and 3MCC deficiency, 1 and 0 case, respectively). In those cases, 12 cases were already treated with some kinds of drugs before onset (vitamin B12-responsive MMA, 4; vitamin B12-unresponsive MMA, 1; PA, 6; HCS deficiency, 1; and $3 \mathrm{MCC}$ deficiency, 0 ). There was no significant difference in the age of onset between MMA and PA patients; however, patients with vitamin B12-responsive MMA had a lower rate $(33 \%)$ of onset within a month of life (data not shown).

We received 116 valid responses concerning the method(s) of diagnosis. There were similar diagnostic tools available in each institution. Nearly all patients $(n=113,97.4 \%)$ underwent analysis of urinary organic acids using gas chromatography mass spectrometry (GC/MS). Other available methods used were tandem mass spectrometry (MS/MS) $(n=38,32.8 \%)$, enzyme activity measurement $(n=47,40.5 \%)$ and genetic analysis $(n=16,13.8 \%)$. Three cases of PA were diagnosed using enzyme activity measurement, genetic analysis and MS/MS without GC/MS. In this study, the detailed results of enzyme activity measurement and genetic analysis could not be collected; however, in previous reports, some kinds of OAs had 
relationship between genotype and phenotype. Table 2 shows the interval time required for diagnosis. Of 97 valid responses, 72 cases $(74.2 \%)$ were diagnosed within a month from onset, 19 cases $(19.6 \%)$ from 1 month to 1 year and 6 cases $(6.2 \%)$ took over a year. These last six cases included two of vitamin B12-unresponsive MMA (one patient died at 12 years of age) and one each of vitamin B12responsive MMA, PA, glutaric aciduria type I and ethylmalonic aciduria.

\section{Clinical characteristics and manifestations}

In MMA and PA, clinical and laboratory findings noted through the patients' clinical course are shown in Table 3a. Most patients had a history of vomiting and appetite loss. In contrast, hypoglycemia and seizure were observed in fewer than $30 \%$ of all cases. Liver dysfunction were found in many cases of vitamin B12-unresponsive MMA $(23 / 42,54.8 \%, P<0.05)$, but fatty liver and hepatomegaly were not statistically significant in this group (17/42, $40.5 \%$ for each). Furthermore, renal dysfunction was more likely to occur in MMA, regardless of the response to vitamin $\mathrm{B} 12$, than in $\mathrm{PA}(P<0.05)$.

Mental retardation is a characteristic clinical feature in OAs. Patients with vitamin B12-unresponsive MMA were significantly more likely to experience mental retardation compared with those with vitamin B12-responsive MMA and PA $(P<0.05)$. Factors associated with mental retardation are shown in Table 3 a. In patients with vitamin B12-unresponsive MMA, factors associated with mental retardation were seizure $(P<0.05)$, liver dysfunction $(P<0.01)$, fatty liver $(P<0.01)$ and hepatomegaly $(P<0.01)$. In patients with vitamin B12-responsive MMA, associated factors were failure to thrive $(P<0.05)$ and seizure $(P<0.05)$. In patients with $\mathrm{PA}$, associated factors were failure to thrive $(P<0.05)$. In addition, liver dysfunction, fatty liver, hepatomegaly and failure to thrive as associated factors were significant indicators regardless of suffering from seizure. Factors associated with mortality are shown in Table 3a. Failure to thrive $(P<0.05)$, pancreatitis $(P<0.05)$ and hypoglycemia $(P<0.01)$ were significantly associated with deceased patients of MMA. The median age of the 10 deceased patients of MMA with failure to thrive was 55 months (IQR, 17-83; range, 2 months to 276 months). Failure to thrive, pancreatitis and hypoglycemia as associated factor with mortality were not significantly related to the maximum value of blood ammonium and blood $\mathrm{pH}$ value at onset and seizure, respectively $(P>0.05)$. The maximum values of blood ammonium at onset are shown in Figure 1a. The median blood ammonium values of vitamin B12-unresponsive MMA $(n=18)$, vitamin B12-responsive MMA $(n=8)$ and PA $(n=10)$ patients were $115 \mu \mathrm{moll}^{-1}$ (IQR, 76343), $103 \mu \mathrm{moll}^{-1}$ (IQR, 74-169) and $120 \mu \mathrm{moll}^{-1}$ (IQR, $72-354$ ), respectively. There was no significant difference in the blood ammonium values between the groups. In cases of mortality and mental retardation, there was no significant difference in the maximum values of blood ammonium at onset among those with MMA. The blood $\mathrm{pH}$ values at onset are shown in Figure $1 \mathrm{~b}$. Within the limit of valid data, the median blood $\mathrm{pH}$ values of vitamin B12unresponsive MMA $(n=18)$, vitamin B12-responsive MMA $(n=7)$ and PA $(n=9)$ patients were 7.15 (IQR, 7.03-7.20), 7.31 (IQR, 7.227.36) and 7.23 (IQR, 7.11-7.37), respectively. Patients with vitamin B12-unresponsive MMA were more likely to have a lower blood $\mathrm{pH}$, without a statistically significant difference, compared with vitamin B12-responsive MMA or PA, but with a statistically significant difference, compared with both vitamin B12-responsive MMA and PA $(P<0.05)$. In cases of mortality and mental retardation, there was no significant change in blood $\mathrm{pH}$ values at the onset of MMA. In
Table 3 The features of clinical and laboratory findings, managements in chronic phase

(a)

\begin{tabular}{|c|c|c|c|}
\hline & $\begin{array}{l}\text { Vitamin B12-unre- } \\
\text { sponsive MMA }\end{array}$ & $\begin{array}{l}\text { Vitamin } B 12- \\
\text { responsive MMA }\end{array}$ & $P A$ \\
\hline Clinical findings (\%) & $N=42$ & $\mathrm{~N}=20$ & $\mathrm{~N}=30$ \\
\hline Vomiting** & $95.2(40 / 42)$ & $70.0(14 / 20)$ & $63.3(19 / 30)^{a}$ \\
\hline Appetite loss** & $95.2(40 / 42)$ & $65.0(13 / 20)$ & $66.7(20 / 30)^{b}$ \\
\hline Seizure & $21.4(9 / 42)^{b}$ & $20.0(4 / 20)^{b}$ & $16.7(5 / 30)$ \\
\hline Mental retardation* & $69.0(29 / 42)$ & $40.0(8 / 20)$ & $43.3(13 / 30)$ \\
\hline Visual impairment & $2.4(1 / 42)$ & $5.0(1 / 20)$ & $6.7(2 / 30)$ \\
\hline Liver dysfunction** & $54.8(23 / 42)^{a}$ & $25.0(5 / 20)$ & $20.0(6 / 30)$ \\
\hline Fatty liver & $40.5(17 / 42)^{a}$ & $10.0(2 / 20)$ & $13.3(4 / 30)$ \\
\hline Hepatomegaly & $40.5(17 / 42)^{a}$ & $10.0(2 / 20)$ & $16.7(5 / 30)$ \\
\hline Renal dysfunction & $35.7(15 / 42)$ & $10.0(2 / 20)$ & $6.7(2 / 30)$ \\
\hline Heart failure & $7.1(3 / 42)$ & $5.0(1 / 20)$ & $10.0(3 / 30)$ \\
\hline Compromised & $7.1(3 / 42)$ & $5.0(1 / 20)$ & 0 \\
\hline Failure to thrive ${ }^{* * c}$ & $69.0(29 / 42)$ & $30.0(6 / 20)^{b}$ & $30.0(9 / 30)^{\mathrm{b}}$ \\
\hline Pancreatitis $^{c}$ & $4.8(2 / 42)$ & 0 & $3.3(1 / 30)$ \\
\hline \multicolumn{4}{|l|}{ Laboratory findings } \\
\hline Hypoglycemia $^{d}$ & $28.6(12 / 42)$ & $10.0(2 / 20)$ & $13.3(4 / 30)$ \\
\hline Hyperuricemia & $31.0(13 / 42)$ & $5.0(1 / 20)$ & $13.3(4 / 30)$ \\
\hline $\begin{array}{l}\text { Neutropenia/ } \\
\text { thrombocytopenia }\end{array}$ & $26.2(11 / 42)$ & $10.0(2 / 20)$ & $13.3(4 / 30)$ \\
\hline
\end{tabular}

(b)

\begin{tabular}{lccc}
\hline & $\begin{array}{c}\text { Vitamin B12-unre- } \\
\text { sponsive MMA }\end{array}$ & $\begin{array}{c}\text { Vitamin B12- } \\
\text { responsive MMA }\end{array}$ & PA \\
Drugs (\%) & $\mathrm{N}=25$ & $\mathrm{~N}=15$ & $\mathrm{~N}=19$ \\
\hline L-carnitine & $100(25 / 25)$ & $86.7(13 / 15)$ & $94.8(18 / 19)$ \\
Arginine & 0 & 0 & $5.3(1 / 19)$ \\
Sodium benzoate & 0 & $6.7(1 / 15)$ & $5.3(1 / 19)$ \\
Vitamin B12 & $28(7 / 25)$ & $93.3(14 / 15)$ & $5.3(1 / 19)$ \\
Metronidazole & $40(10 / 25)$ & $6.7(1 / 15)$ & $5.3(1 / 19)$ \\
Urinary alkalizer & $56(14 / 25)$ & $6.7(1 / 15)$ & $15.8(3 / 19)$ \\
& & & \\
Low-protein diet $(\%)$ & $88(22 / 25)$ & $38.5(5 / 13)$ & $46.7(7 / 15)$ \\
$\quad$ At 2 weeks after & & & \\
onset & & & \\
$\quad$ Chronic phase & $93(14 / 15)$ & $25(3 / 12)$ & $50(8 / 16)$
\end{tabular}

Independence was tested by chi-square test. On small numbers, data were analyzed by Fisher exact test for a more precise $P$-value.

Related factor to mental retardation on MMA, PA $(P<0.01)$

bRelated factor to mental retardation on MMA, PA $(P<0.05)$

cRelated factor to mortality on overall MMA $(P<0.05)$.

dRelated factor to mortality on overall MMA $(P<0.01)$.

eUrinary alkalizer: ularyt, sodium bicarbonate.

${ }^{*} P<0.05,{ }^{* *} P<0.01$.

addition, there was no significance in outcomes between mental retardation and blood $\mathrm{pH}$ values at onset.

\section{Treatment and outcome}

We investigated the required intensive care treatments, such as hemodialysis, exchange transfusion, peritoneal dialysis, respirator use and catecholamine administration, during the acute phase. In total, $40.0 \%(12 / 30), 13.3 \%(2 / 15)$ and $23.8 \%(5 / 21)$ of patients with vitamin B12-unresponsive MMA, vitamin B12-responsive MMA and $\mathrm{PA}$, respectively, required intensive care treatments. Vitamin B12- 

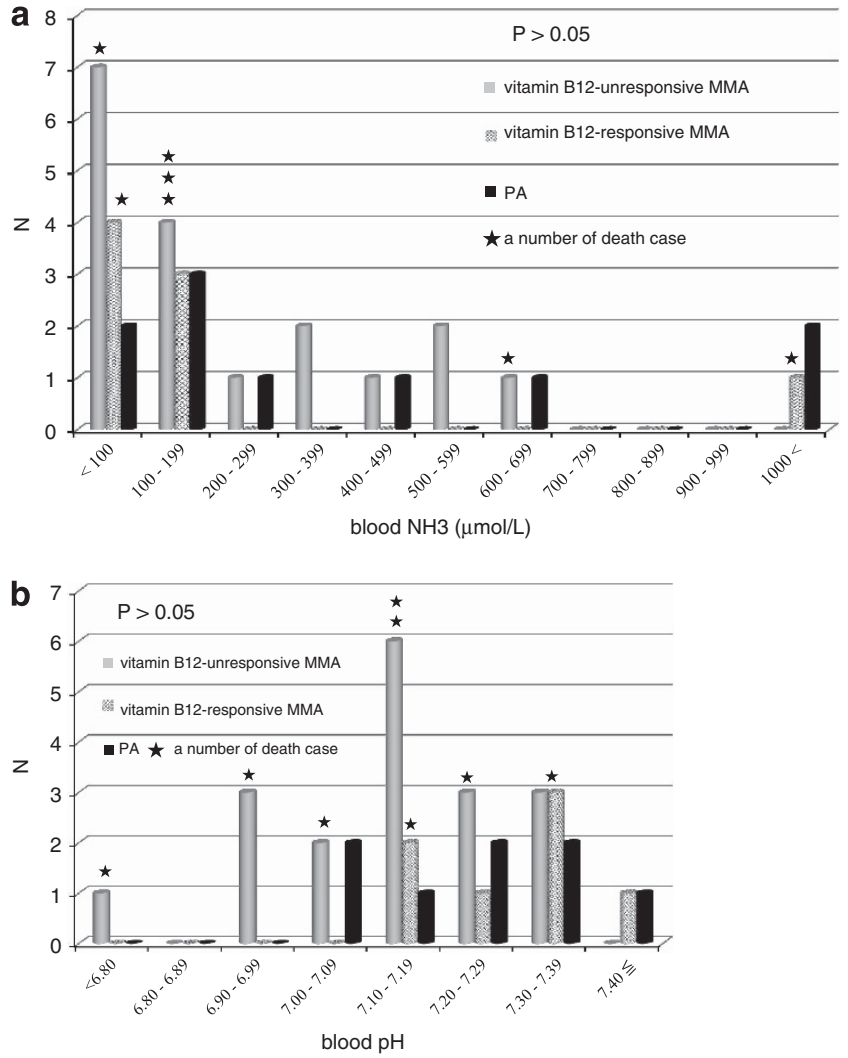

Figure 1 Histogram of methylmalonic acidemia (MMA) and propionic acidemia (PA) patients with different blood ammonium values $\left(\mu \mathrm{mol} \mathrm{I}^{-1}\right)(\mathrm{a})$ and blood $\mathrm{pH}$ values (b) at onset. (a) Histogram of MMA and PA patients with different blood ammonium values $\left(\mu \mathrm{moll}^{-1}\right)$ at onset. A total of 36 patients had available data. Over 50\% had blood ammonium levels $<200 \mu \mathrm{moll}^{-1}$. Most of the deceased patients did not have significantly high blood ammonium levels $(P>0.05)$. There was no significance between the groups, after analysis by the Kruskal-Wallis test $(P>0.05)$. (b) Histogram of MMA and PA patients with different blood $\mathrm{pH}$ values at onset. A total of 33 patients had available data. Vitamin B12-unresponsive MMA patients had lower blood $\mathrm{pH}$ compared with vitamin B12-responsive MMA and PA patients. Blood $\mathrm{pH}$ values did not have relationship between the deceased MMA patients and survived MMA patients $(P>0.05)$. There was no significance between groups on analysis with the Kruskal-Wallis test $(P>0.05)$.

unresponsive MMA patients were significantly more likely to undergo intensive care treatment $(P<0.05)$. In these patients, hemodialysis was performed in $16.7 \%(5 / 30)$, exchange transfusion in $16.7 \%(5 /$ $30)$, peritoneal dialysis in $20.0 \%(6 / 30)$, respirator use in $20.0 \%(6 / 30)$ and catecholamine administration in $17.2 \%$ (5/30) of cases. Among all the OAs, deceased patients required hemodialysis, exchange transfusion or peritoneal dialysis significantly $(P<0.05)$.

Table $3 \mathrm{~b}$ shows the management for the chronic phase, including the administration of medications and a low-protein diet. L-Carnitine was prescribed for almost all patients (94.9\%) of MMA and PA. Arginine and sodium benzoate was seldom prescribed for MMA and PA patients $(0 \%, 2.5 \%$ for MMA and $5.3 \%, 5.3 \%$ for PA, respectively). Vitamin B12-responsive MMA patients were treated with vitamin B12, except for one patient who underwent liver transplantation. Metronidazole and a combination of sodium bicarbonate and citrate were more often prescribed to vitamin B12unresponsive MMA patients at rates of $40 \%$ and $56 \%$, respectively, compared with vitamin B12-responsive MMA and PA. With regard to

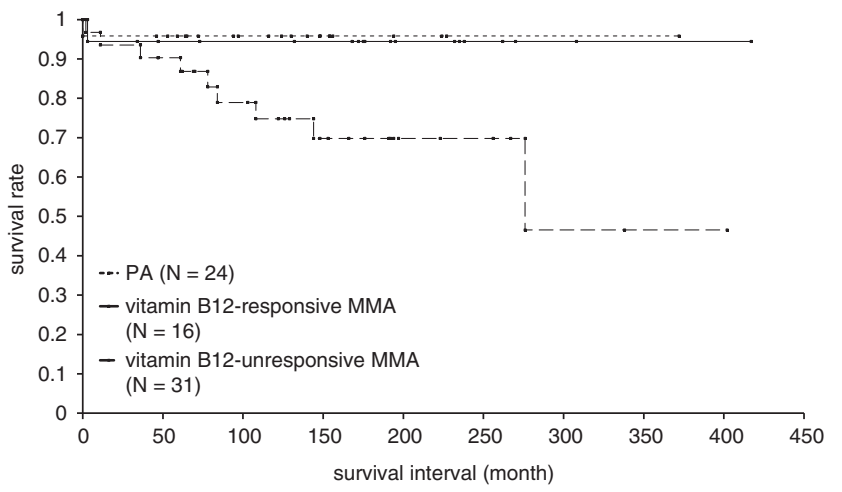

Figure 2 Survival rates in patients with methylmalonic acidemia (MMA) and propionic acidemia (PA). The survival rates of MMA and PA patients without liver transplantation are illustrated as Kaplan-Maier curves. The survival rates of $\mathrm{PA}$, vitamin B12-responsive MMA and vitamin B12-unresponsive MMA patients at 20 years of age were $95.8,94.4$ and $69.8 \%$, respectively. Significant values were revealed by logrank testing between groups $(P<0.05)$.

nutrition management, we assessed the administration of a restricted protein diet at 2 weeks after onset and during the chronic phase (Table 3b). Vitamin B12-unresponsive MMA patients were given a strictly restricted protein diet, in comparison with vitamin B12responsive MMA and PA patients. During the chronic phase, feeding support, such as a naso-gastric tube and gastrostomy tube, was required in $40.0 \%$ of vitamin B12-unresponsive MMA, $20.0 \%$ of vitamin B12-responsive MMA and $23.5 \%$ of PA patients. The survival rate was compared between MMA and PA using the Kaplan-Meier curves (Figure 2). There was valid data for the outcomes of 31 vitamin B12-unresponsive MMA, 16 vitamin B12-responsive MMA and $24 \mathrm{PA}$ patients. Ten vitamin B12-unresponsive MMA patients died due to severe acidosis $(n=3)$, cardiomyopathy $(n=1)$ and unknown causes $(n=6)$. Of the 65 MMA patients with valid data regarding liver transplantation, 13 received liver transplantation (11 vitamin B12-unresponsive MMA patients and 2 vitamin B12-responsive MMA patients). In addition, six PA patients underwent liver transplantation. As the number of liver transplantation cases was limited in this study, the survival rate was estimated in nontransplanted cases. The survival rate of vitamin B12-unresponsive MMA was significantly lower than those of vitamin B12-responsive MMA and PA. In vitamin B12-unresponsive MMA patients, the survival rate gradually decreased to $69.8 \%$ by the age of 144 months, following which the survival rate was steady until 276 months. Moreover, the survival rate at 10 years of age in the early-onset type and the late-onset type of vitamin B12-unresponsive MMA were $61.0 \%$ and $81.0 \%$, respectively, which was no significant difference between the types. In contrast, the survival rates at 20 years of age in vitamin B12-responsive MMA and PA patients were 94.4\% and $95.8 \%$, respectively.

\section{DISCUSSION}

OAs are often life-threatening immediately after disease onset and can result in severe sequelae. ${ }^{1}$ These rare diseases require further investigation to improve the current management and outcomes of OAs. This study revealed the clinical features of OAs in Japan, especially in cases with MMA and PA. We found that the majority of patients with OAs had MMA, followed by PA, together accounting for $\sim 80 \%$ of all OAs in Japan. 
There are several limitations to this study. First, most patient information available was restricted to neonates, infants and children. Second, tandem mass spectrometry (MS/MS) was not used as a general screening tool in all OAs. Since the early 1990s, MS/MS has been considered an effective tool for diagnosing inborn errors of metabolism. ${ }^{4,10,11}$ In Japan, a pilot study examining the use of MS/MS was started in 1997; however, MS/MS is just becoming a part of government screening in recent years. In the present study, 15 cases of OAs were diagnosed by NBS using MS/MS or advanced metabolic tests before onset. Seven possible mild PA patients could be involved in this study. Third, there may have been some bias as most patients with OAs were managed by specialists in inherited metabolic diseases, and therefore might have good outcomes. It is possible that there were some patients who received limited treatment and/or management and others who were not diagnosed; however, in Japan, patients with OAs are typically referred to specialists in inherited metabolic diseases.

\section{Blood glucose as an important indicator of a poor prognosis in MMA and PA}

Previous studies report that the majority of MMA and PA patients were diagnosed in the neonatal period. ${ }^{12-15}$ In the present study, the majority of MMA and PA patients had an early onset, and almost all of the remaining patients manifested clinical signs within the first year of life. OAs are characterized by common symptoms, possibly leading to decompensation. ${ }^{1,15}$ The present study indicated a significant relationship between hypoglycemia and mortality in vitamin B12unresponsive MMA patients. Hypoglycemia was not a common feature of MMA and PA, but it was a significant prognostic factor of mortality in vitamin B12-unresponsive MMA patients. Hypoglycemia might be induced by graduated severity of decompensation because it was the results of decompensation regarding glucose metabolism. Furthermore, hyperglycemia in MMA patients was also indicative of a poor prognosis. ${ }^{16,17}$ Thus, for careful monitoring of MMA and PA, blood glucose is an important marker. ${ }^{17}$

\section{Difference in the management of patients with MMA and PA}

Aggressive, broad therapy is often necessary to treat OAs at onset because of the possibility of severe sequelae and death. Affected individuals should be referred to a metabolic center where intensive care is available. ${ }^{18-20}$ In the present study, the frequency of intensive care treatment was greater in MMA patients than in PA patients. Hemodialysis, exchange transfusion or peritoneal dialysis were used significantly in all mortality cases. Furthermore, in the present study, L-carnitine was prescribed for almost all affected individuals, which is similar to the recommended treatment. ${ }^{14,19,21}$ The importance of preventing secondary carnitine deficiency is relatively well understood in the management of OAs. Moreover, it is important to intake adequate amount of protein while managing nutrition. ${ }^{22}$ When affected patients are in a state of decompensation, a strict restrictive protein diet is necessary for recovery. However, after recovery from the decompensation, infants and children require sufficient protein for growth. In the present study, many vitamin B12-unresponsive MMA patients were given a restricted protein diet, and $70 \%$ of them were found to have failure to thrive, which is likely a result of the limited diet.

Clinical features associated with mental retardation and mortality in MMA and PA

Failure to thrive was associated with mortality in patients with MMA. Because the 10 deceased patients of MMA with failure to thrive involved three infants under the 12 months of age, failure to thrive was important factor to management for not only after infant period but also during infant period. Furthermore, failure to thrive as associated factor with mortality was not related to blood ammonium value and $\mathrm{pH}$ value at onset, and seizure. Vitamin B12-responsive MMA and PA patients received a restricted protein diet less frequently than vitamin B12-unresponsive MMA. However, failure to thrive was significantly related to mental retardation in vitamin B12-responsive MMA and PA patients. In MMA patients, seizures were associated with mental retardation regardless of the response to vitamin B12, similarly to a previous report. ${ }^{23}$ Moreover, liver dysfunction, hepatomegaly and fatty liver were related to mental retardation in vitamin B12-unresponsive MMA patients, but not to mortality. In addition, these associated factors to mental retardation were indicators regardless of suffering from seizure. In this study, because of limited valid data, influence of the blood ammonium value and $\mathrm{pH}$ value at onset on the clinical feature as associated factors to mental retardation was unclear. In PA patients, vomiting and appetite loss were related to mental retardation significantly. However, those were common and nonspecific symptoms in metabolic disease.

The majority of deaths occurred in vitamin B12-unresponsive MMA patients. Therefore, there was significant difference in mortality rates between vitamin B12-unresponsive MMA, vitamin B12-responsive MMA and PA patients. Concerning treatment of OAs, more MMA patients required intensive care treatment compared with PA patients, especially hemodialysis, exchange transfusion or peritoneal dialysis were used significantly in all mortality cases. There was no significance found in blood ammonium values at the onset in MMA and PA patients. In comparison, progressive acidosis was observed in vitamin B12-unresponsive MMA patients, who had significantly lower blood $\mathrm{pH}$ compared with patients with vitamin B12-responsive MMA and PA $(P<0.05)$. This was likely due to the effects of toxic metabolites such as methylmalonate on secondary mitochondrial dysfunction, as previously reported. ${ }^{15,24}$

\section{Outcome}

We analyzed the outcomes of MMA and PA patients using KaplanMaier curves (Figure 2). Because of improving therapies including nutrition management, drugs and hemodialysis, the survival rates of MMA and PA patients have increased in comparison with previous reports. In the present study, there was a significant difference in mortality among vitamin B12-unresponsive MMA, vitamin B12-responsive MMA and PA patients. The survival rate in vitamin B12-unresponsive MMA patients gradually decreased to $69.8 \%$ by the age of 144 months. In contrast, that in PA patients at 20 years of the age was $95.8 \%$. MMA was much severe than PA, but clinically classical PA was more severe than or at least as severe as vitamin B12-unresponsive MMA. In this study, though the survival rate in PA patients was better than that of vitamin B12-unresponsive MMA, PA patients could involve seven possible mild PA patients. Those possible mild form PA patients might have the mild form specific mutation (Y435C) in the PCC gene and might not develop acute decompensation. ${ }^{25}$ Because of including mild PA patients in analysis, the survival rate of PA might be higher than that excluding mild PA patients. In the future, as the use of NBS with MS/MS increases, it is our hope that the mortality rates of MMA and PA patients will continue to decrease as previously described. ${ }^{5}$ Furthermore, factors associated with mental retardation and death may affect the indications for liver transplantation. 


\section{CONCLUSION}

Patients with vitamin B12-unresponsive MMA were more likely to develop severe manifestations and complications than those with vitamin B12-responsive MMA and PA. Failure to thrive, hypoglycemia and pancreatitis associated with mortality are amenable factors to appropriate management, especially in cases with vitamin B12unresponsive MMA. The survival rates at 20 years of age in vitamin B12-unresponsive MMA, vitamin B12-responsive MMA and PA patients were $69.8 \%, 94.4 \%$ and $95.8 \%$, respectively.

\section{ACKNOWLEDGEMENTS}

This study was supported in part by a Grant-in-Aid for the Global COE Program from the Japanese Society for the Promotion of Science and Ministry of Education, Culture, Sports, Science and Technology; a Grant-in-Aid for Pediatrics Research from the Ministry of Health, Labor and Welfare; and a Grant-in-Aid for Scientific Research from the Ministry of Education, Culture, Sports, Science and Technology. We thank all the participants for their cooperation in this study.

1 Fenton, W. A., Gravel, R. A. \& Rosenblatt, D. S. in The Metabolic and Molecular Bases of Inherited Disease 8th (eds Scriver, C. R., Beaudet, A. L., Sly, W. S. \& Valle, D.) 2165-2193 (McGraw-Hill, New York, 2001).

2 Kido, J., Nakamura, K., Mitsubuchi, H., Ohura, T., Takayanagi, M., Matsuo, M. et al. Long-term outcome and intervention of urea cycle disorders in Japan. J. Inherit. Metab. Dis. 35, 777-785 (2012).

3 Kido, J., Nakamura, K., Matsumoto, S., Mitsubuchi, H., Ohura, T., Shigematsu, Y. et al. Current status of hepatic glycogen storage disease in Japan: clinical manifestations, treatments and long-term outcomes. J. Hum. Genet. 58, 285-292 (2013).

4 Millington, D. S., Kodo, N., Norwood, D. L. \& Roe, C. R. Tandem mass spectrometry: a new method for acylcarnitine profiling with potential for neonatal screening for inborn errors of metabolism. J. Inherit. Metab. Dis. 13, 321-324 (1990).

5 Grünert, S. C., Müllerleile, S., de Silva, L., Barth, M., Walter, M., Walter, K. et al. Propionic acidemia: neonatal versus selective metabolic screening. J. Inherit. Metab. Dis. 35, 41-49 (2012).

6 Shigemastu, Y., Hirano, S., Hata, I., Tanaka, Y., Sudo, M., Sakura, N. et al. Newborn mass screening and selective screening using electrospray tandem mass spectrometry in japan. J. Chromatogr. B Analyt. Technol. Biomed. Life Sci. 776, 39-48 (2002).

7 Yamaguchi, S. Newborn screening in Japan: restructuring for the new Era. Ann. Acad. Med. Singapore 37, 13-17 (2008).

8 Shigeamatsu, Y., Hata, I., Kikawa, Y., Mayumi, M., Tanaka, Y., Sudo, M. et al. Modifications in electrospray tandem mass spectrometry for a neonatal-screening pilot study in Japan. J. Chromatogr. B Biomed. Sci. Appl. 731, 97 (1999).
9 Zwicker, T., Lindner, M., Aydin, H. I., Baumgartner, M. R., Bodamer, O. A., Burlina, A. B. et al. diagnostic work-up and management of patients with isolated methylmalonic acidurias in European metabolic centers. J. Inherit. Metab. Dis. 31, 361-367 (2008).

10 Klose, D. A., Kölker, S., Heinrich, B., Prietsch, V., Mayatepek, E., von Kries, R. et al. Incidence and short-term outcome of children with symptomatic presentation of organic acid and fatty acid oxidation disorders in Germany. Pediatrics 110, 1204-1211 (2002)

11 Dionisi-Vici, C., Deodato, F., Röschinger, W., Rhead, W. \& Wilcken, B. 'Classical organic acid, propionic acidurias, methylmalonic acidurias and isovaleric acidurias: long-term outcome and effects of expanded newborn screening using tandem mass spectrometry. J. Inherit. Metab. Dis. 29, 383-389 (2006).

12 Cosson, M. A., Benoist, J. F., Touati, G., Déchaux, M., Royer, N., Grandin, L. et al. Long-term outcome in methylmalonic aciduria:A series of 30 French patients. Mol. Genet. Metab. 97, 172-178 (2009).

13 van der Meer, S. B., Poggi, F., Spada, M., Bonnefont, J. P., Ogier, H., Hubert, P. et al Clinical outcome of long-term management of patients with vitamin B12-unresponsive methylmalonic acidemia. J. Pediatr. 125, 903-908 (1994).

14 Grünert, S. C., Müllerleile, S., De Silva, L., Barth, M., Walter, M., Walter, K. et al. Propionic acidemia: clinical and outcome in 55 patients and adolescent patients. Orphannet. J. Rare Dis. 8, 6 (2013).

15 Pena, L. \& Burton, B. K. Survey of health status and complications among propionic acidemia patients. Am. J. Med. Genet. 158A, 1641-1646 (2012).

16 Boeckx, R. L. \& Hicks, J. M. Methylmalonic acidemia with the unusual complication of severe hyperglycemia. Clin. Chem. 28, 1801-1803 (1982).

17 Imen, M., Hanene, B., Ichraf, K., Aida, R., Ilhem, T., Naziha, K. et al. Methylmalonic acidemia and hyperglycemia: an unusual association. Brain. Dev. 34, 113-114 (2012)

18 Lee, N. C., Chien, Y. H., Peng, S. F., Huang, A. C., Liu, T. T., Wu, A. S. et al. Brain damage by mild metabolic derangements in methylmalonic academia. Pediatr. Neurol. 39, 325-329 (2008).

19 Sutton, V. R. Chapman, K. A. Gropman, A. L., MacLeod, E., Stagni, K., Summar, M. L. et al. Chronic management and health supervision of individuals with propionic acidemia. Mol. Genet. Metab. 105, 26-33 (2012).

20 Chapman, K. A., Gropman, A., MacLeod, E., Stagni, K., Summar, M. L., Ueda, K. et al. Acute management of propionic acidemia. Mol. Genet. Metab. 105, 16-25 (2012).

21 Deodato, F., Boenzi, S., Santorelli, F. M. \& Dionisi-Vici, C. Methylmalonic and propionic aciduria. Am. J. Med. Genet. 142C, 104-112 (2006).

22 Yannicelli, S. Nutrition therapy of organic acidaemias with amino-based formulas: emphasis on methylmalonic and propionic acidemia. J. Inherit. Metab. Dis. 29, 281-287 (2006)

23 O'Shea, C. J., Sloan, J. L., Wiggs, E. A., Pao, M., Gropman, A., Baker, E. H. et al. Neurocognitive phenotype of isolated methylmalonic academia. Pediatrics 129 , e1541-e1551 (2012)

24 Melo, D. R., Mirandola, S. R., Assunção, N. A. \& Castilho, R. F. Methylmolonate impairs mitochondrial respiratory supported by NADH-linked substrates: involvement of mitochondrial glutamate metabolism. J. Neurosci. Res. 90, 1190-1199 (2012).

25 Yorifuji, T., Kawai, M., Muroi, J., Mamada, M., Kurokawa, K., Shigematsu, Y. et al. Unexpectedly high prevalence of the mild form of propionic acidemia in Japan: presence of a common mutation and possible clinical implications. Hum. Genet. 111, 161-165 (2002) 